\title{
REWORKING A SIMPLE MODEL FOR DEFORMABLE PROJECTILE PENETRATION INTO THICK TARGETS
}

\author{
STAVROS SYNGELLAKIS \\ Wessex Institute of Technology, UK
}

\begin{abstract}
Target penetration by deformable projectiles is considered in the context of an established onedimensional theory based on the fundamental balance laws of continuum mechanics. The paper critically reviews the assumptions of this simple analytical model and discusses the material characteristics that influence resistance to penetration. A novel representation of the resisting force is introduced into the original analysis leading to the evaluation of the crater size in addition to other critical output such as depth of penetration and the time history of the impact force over a relevant range of impact energies. An algorithm for a time step integration of the resulting system of differential equations is proposed; this is numerically implemented through a FORTRAN code whose effectiveness and reliability are tested against experimental data from the technical literature. This comparison leads to rational and consistent estimates for the empirical factors adopted in the simplified modelling. The time histories of impulse provided by the computer implemented analysis can be used for the assessment of the effect of the transmitted shock on the supporting structure and its appendices.
\end{abstract}

Keywords: deformable projectile, long rod, semi-infinite target, penetration, high impact velocity, onedimensional model.

\section{INTRODUCTION}

Protective plating can be designed to sustain locally high-energy impact. However, high penetration resistance means that a large amount of impact energy is transmitted to the protected structure and may have a detrimental effect on the plating supports as well as any sensitive equipment within the structure. It is thus important to identify the intensity and duration of the shock in order to assess its effect on vulnerable elements.

The phenomena arising from projectile impact vary considerably with the type, range and intensity of attack, as well as projectile and target geometry and material properties. Projectiles relying only on their kinetic energy have been designed in various shapes and sizes with the purpose of optimizing their penetration performance. The focus in this paper is on slender projectiles, which are expected to deform plastically upon impact and gradually erode during penetration as a result of the high impact velocity range and projectile-target strength combinations considered. It is reasonable to idealize thick targets as semi-infinite media and assess their response under normal projectile incidence.

The problem has often been addressed through test data correlation yielding empirical relations between ballistic parameters and impact velocity, geometry and material properties [1]. This approach, apart from its cost due to the required extensive experimentation, also provides output of limited scope. Finite element and finite difference solutions based on continuum mechanics theories yield complete answers and thus valuable insight into the impact phenomena [2]-[4], but these numerical simulations can be computationally expensive and time-consuming.

In this paper, project penetration is modelled as a one-dimensional problem formulated by applying fundamental physical laws combined with rational simplifying assumptions supported by experimental evidence. Such simple models, if properly validated, are reliable, economical and applicable to specific impact cases or ranges of parameters. They quickly 
provide time histories of impact loading and penetration which can be used for the assessment of overall structural response and the design of impact experiments.

The development of simple penetration theories for deformable projectiles has a long history, which has been reviewed, to a greater or lesser extent, in previous papers addressing this problem. Recent work in this area includes that by Rubin and Yarin [5] who developed a simple formula for predicting penetration depth and $\mathrm{Lu}$ and Wen [6] who produced a detailed and comprehensive theory encompassing deformable projectile penetration over a wide range of impact velocities.

The basis of the present analysis is the version of a model presented by Wright and Frank [7] who also provided a fairly comprehensive review of related earlier work. The scope of that analysis is here enhanced by combining it with a recently proposed model for the target dynamic pressure on hemispherical target-projectile interfaces [8]. According to this model, the penetration process is governed by a system of first-order differential equations. The numerical integration scheme adopted for its solution allows for non-uniform projectile cross-section thus widening the range of the method's applicability. The model includes physical parameters whose values are not easily identifiable through testing and have therefore to be assumed. Values of empirical constants appearing in the governing equations are identified by comparing qualitatively and quantitatively predictions by the proposed model with previously published experimental data and other analytical findings. Thus, time histories of all dynamic variables involved can be readily and reliably obtained.

\section{BACKGROUND THEORY}

\subsection{Assumptions}

The impact of projectiles onto thick targets has been observed first to cause shock wave propagation, then quasi-steady flow fields and finally either failure of target material resulting in perforation or termination of penetration and rapid transition to projectile rest. It has been argued that the second stage dominates the deformation process in the case of long, slender projectiles [9]. Its mathematical modelling through a simple one-dimensional theory is presented in this section. The numerical implementation of this theory can approximately provide the time profile and duration of impulse.

The projectile, in the shape of an axisymmetric long rod, is eroded during penetration. Normal incidence on the semi-infinite medium is assumed as this is expected to cause maximum damage for a given impact energy. Fig. 1 shows diagrammatically the steady-state penetration phase of the projectile into a thick target. The rod is considered comprising three parts: a gradually diminishing rigid one (region R), a constant volume one under steady-state plastic flow (region $\mathrm{S}$ ) and a rearwards flowing debris (region D).

\subsection{Conservation laws}

As mentioned in the Introduction, the equations governing the projectile motion are derived according to the process adopted by Wright and Frank [7]. A consistent system of equations is formulated by applying the conservation laws of mechanics. As is evident in Fig. 1, the boundaries move relative to the material, it is, therefore, essential to include in the constitutive equations the terms due to material convection.

First, conservation of mass and linear momentum applied to the eroded rod leads to the differential equations

$$
\dot{V}_{R}(x)=A_{R}(x)(\dot{x}-u),
$$




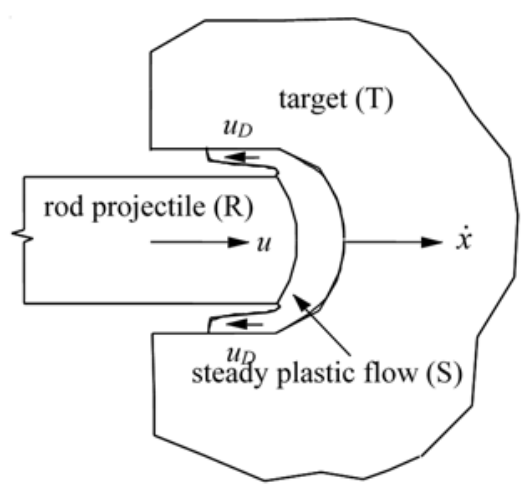

Figure 1: Sketch of long projectile penetrating a thick target.

$$
V_{R} \dot{u}=-\frac{A_{R} \sigma_{f R}}{\rho_{R}},
$$

where $x$ is the penetration depth, $u(t), V_{R}(x, t)$ and $A_{R}(x, t)$ are, respectively, the velocity, volume and front area of the rigid part of the rod at any time during penetration, $\rho_{R}$ its density, $\sigma_{f R}$ its dynamic flow stress, subscript $R$ indicates a rigid rod item and a dot over a symbol indicates differentiation of the represented quantity with respect to time. This form of the equations allows consideration of projectiles with long pointing heads such as cones or ogives.

In the case of rods with uniform cross-section, eqns (1) and (2) are simplified to

$$
\begin{gathered}
\dot{L}=\dot{x}-u, \\
L \dot{u}=-\frac{\sigma_{f R}}{\rho_{R}},
\end{gathered}
$$

where $L(t)$ is the current length of the eroded rod. It is easily shown that conservation of energy is identically satisfied if eqns (1) and (2) or (3) and (4) are satisfied.

Next, conservation of mass, momentum and energy are applied to the constant-volume plastic material in steady-state motion. This leads to the equations

$$
\begin{gathered}
0=\rho_{R} A_{R}(-\dot{x}+u)+\rho_{D} A_{D}\left(-\dot{x}-u_{D}\right), \\
0=\rho_{R} A_{R} u(-\dot{x}+u)-\rho_{D} A_{D} u_{D}\left(-\dot{x}-u_{D}\right)+F_{R}+F_{D}-F_{T}, \\
0=\rho_{R} A_{R}\left(e_{R}+1 / 2 u^{2}\right)(-\dot{x}+u)+\rho_{D} A_{D}\left(e_{D}+1 / 2 u_{D}^{2}\right)\left(-\dot{x}-u_{D}\right)+\dot{W},
\end{gathered}
$$

where subscripts $D$ and $T$ refer to debris and target regions, respectively, $A$ represents interface areas, $F$ interface forces, $e$ internal energy densities and

$$
\dot{W}=\int_{R S} \mathbf{n} \cdot \mathbf{t} \cdot \mathbf{u} d S+\int_{D S} \mathbf{n} \cdot \mathbf{t} \cdot \mathbf{u} d S+\int_{S T} \mathbf{n} \cdot \mathbf{t} \cdot \mathbf{u} d S=F_{R} u-F_{D} u_{D}-F_{T} \dot{x}+\dot{W}_{S T}^{\text {shear }}
$$

is the rate of work done by interface forces. 
The RS and DS interfaces are assumed approximately flat, but ST is considered curved thus the last term on the right-hand side of eqn (8) represents the rate of work by shearing traction over that interface. Interface forces can be expressed in terms of average interface stresses by

$$
F_{R}=A_{R} \sigma_{f R}, \quad F_{D}=A_{D} \sigma_{D}
$$

Mathematical manipulation of eqns (5)-(7), accounting for eqn (8), leads to the expressions

$$
\begin{aligned}
& 2 \rho_{R}(-\dot{x}+u)^{2}=\frac{p_{e}^{2}}{p_{e}-\sigma_{e}}, \\
& 2 \rho_{R}\left(\dot{x}+u_{D}\right)^{2}=\frac{\left(p_{e}-2 \sigma_{e}\right)^{2}}{p_{e}-\sigma_{e}},
\end{aligned}
$$

with the definitions

$$
\begin{gathered}
p_{e}=-\sigma_{f R}-\frac{A_{D}}{A_{R}} \sigma_{D}+\frac{F_{T}}{A_{R}}, \\
\sigma_{e}=\rho_{R}\left(e_{D}-e_{R}\right)-\sigma_{f R}+\frac{\rho_{R}}{\rho_{D}} \sigma_{D},
\end{gathered}
$$

The rate of work of shearing tractions over the ST interface was assumed negligible due to either local melting or adiabatic shear [7]. The right-hand sides of eqns (9) and (10) can be further simplified by accounting for the following assumptions [7]:

- $\quad$ The rod is rigid, hence $e_{R} \cong 0$.

- Debris material is highly fragmented, hence $F_{D}=A_{D} \sigma_{D} \cong 0$.

- Heating and fracture energy is accounted for by the term $\rho_{R} e_{D}$, which can then be assumed of the same order of magnitude as $\sigma_{f R}$.

These assumptions lead to the hypothesis that $\sigma_{e}$ is small compared to $p_{e}$ and the approximations

$$
\begin{aligned}
& 2 \rho_{R}(-\dot{x}+u)^{2} \cong p_{e}+\sigma_{e}, \\
& 2 \rho_{R}\left(\dot{x}+u_{D}\right)^{2} \cong p_{e}-3 \sigma_{e} .
\end{aligned}
$$

\section{NEW FORMULATION}

\subsection{Projectile-target interaction}

The theory comprising eqns (3)-(5), (13) and (14) needs to be complemented by a model for the resisting force $F_{T}$ acting on the ST interface. Various expressions have been proposed for that force or the interface pressure, most of them in the context of a rigid projectile penetration analysis. Such formulae usually comprise a constant term and other terms depending on penetration velocity and acceleration [1]. In the present paper, the form proposed by Hill [10], which accounts for the shape of the projectile-target interface, is adopted, namely,

$$
F_{T}=\lambda^{2} A_{T} a \sigma_{F},
$$


where $\lambda$ is the ratio of the crater to projectile diameter depending on the penetration velocity, $\varphi_{T}$ the target dynamic flow stress and $a$ an empirically determined factor. According to Hill's model, cavitation occurs for penetration velocities above a critical value, the cavitation velocity.

It is reasonable to expect that a deformable projectile in hypervelocity impact would behave like a blunt-nose penetrator. Previous simplified [11] as well as numerical [12] analyses have actually yielded an elliptical projectile-target interface. The application of eqn (15) to the present problem is here based on the assumption that the ST interface is approximately hemispherical and the projected area of this hemispherical head is given by

$$
A_{T}=A_{R}+A_{D}
$$

Recently, an improved version of Hill's model was developed for hemispherical heads [8] according to which

$$
\lambda(\dot{x})=\frac{1+\alpha^{2}}{2 \alpha}, \alpha=\frac{\dot{x}}{\dot{x}_{c}},
$$

with the cavitation velocity given by

$$
\dot{x}_{c}=\left(\frac{a \sigma_{f T}}{K \rho_{T}}\right)^{1 / 2},
$$

where $K$ is another empirical factor determined experimentally.

For high penetration velocities, the new model for the resisting force $F_{T}$ predicts partial contact between projectile head and target; it is thus consistent with relevant numerical work [12], which revealed high pressure concentration over the central portion of projectile-target interface. It also provides a prediction for the cavity which, as test results [13] and numerical work [14] have shown, depends strongly on penetration velocity and can become as large as twenty times the projectile cross-sectional area at impact velocities above $3 \mathrm{~km} / \mathrm{s}$.

Substituting the adopted expression for $F_{T}$, eqn (15), into the expression for $p_{e}$, eqn (11), and accounting for all the other assumptions made above changes eqns (13) and (14) to the following forms:

$$
\begin{aligned}
& 2 \rho_{R}(-\dot{x}+u)^{2}=\lambda^{2}(\dot{x})\left(1+\frac{A_{D}}{A_{R}}\right) a \sigma_{f T}-2 \sigma_{f R}+\rho_{R} e_{D}, \\
& 2 \rho_{R}\left(\dot{x}+u_{D}\right)^{2}=\lambda^{2}(\dot{x})\left(1+\frac{A_{D}}{A_{R}}\right) a \sigma_{f T}+2 \sigma_{f R}-3 \rho_{R} e_{D} .
\end{aligned}
$$

\subsection{Values of model parameters}

Numerical answers obtained from the outlined theory require rational input for the various material parameters and empirical constants appearing in the model. Starting from the flow stresses, their values depend on plastic strain, which is itself space- and time-dependent but since both projectile and target are locally at an advanced stage of plastic deformation, it is reasonable to adopt the maximum tensile strength as the flow stress in the calculations. This choice is also justified by the observation that significant plastic strain develops even in low- 
energy impacts [15]. The value of the tensile strength is easily identifiable for most materials from both printed and internet sources. It is also empirically related to Brinell hardness [16], which is often given for the tested materials [13].

As already mentioned, $\rho_{R} e_{D}$ and $\sigma_{f R}$ must be of the same order of magnitude; this conclusion is reached by considering realistic temperature rises and accounting for the heat capacity of the projectile material [5]. Because of the uncertainty regarding a rational value for $e_{D}$, its magnitude is here controlled by a parameter $\beta$, introduced into the model through the relation

$$
\rho_{R} e_{D}=\beta \sigma_{f R}
$$

In computations, a search for an appropriate value for the factor $b$ is undertaken, guided by the consistency between analytical output and experimental measurements. This comparison clearly indicated a degree of dependence of $\beta$ on the impact energy, especially in the lower impact velocity range. For this reason, a simple relation for $\beta$ was adopted for penetration velocities below the cavitation one, namely

$$
\beta=\frac{\dot{x}}{\dot{x}_{c}} \beta_{0},
$$

where $\beta_{0}$ is the constant $\beta$-value identified for velocities higher that the cavitation one.

Parameter $K$ has been defined and discussed in previous articles on rigid projectile penetration [8], [10]. It is a dimensionless, positive parameter, playing the role of a shape drag coefficient; as such, it is expected to depend predominantly on head shape and this was confirmed experimentally. For hemispherical heads, in particular, results from a finite element simulation of penetration [17] led to a value of $K$ around 3.4. Comparison of the simple, rigid projectile analysis predictions with experimental data gave a value for $K$ of around 3.3 [8]. Due to this consistency, the value $K=3.5$ was adopted in all applications of the theory presented in this paper.

Parameter $a$ basically accounts for the kinematic constraint on plastic deformation in the neighbourhood of the impact zone. Its value does therefore depend mainly on the plastic properties of the projectile-target materials. At high impact velocity, the projectile produces a tunnel through the target material and therefore it has been proposed [7] that $a$ is obtained from elasto-plastic solutions for the pressure causing cylindrical or spherical cavity expansions [18]. For rigid projectiles with hemispherical head, numerical analysis results [17] suggest a value of $a$ around 4 [8] but this may not be relevant to deformable projectile penetration. Thus, it was decided that, as with parameter $\beta$, a reliable estimate of $a$ will be obtained by comparing the results of the present analysis with experimental data.

\subsection{Solution algorithm}

A numerical integration scheme was required for the solution of the system of coupled first order differential eqns (3), (4) and (18). Eqns (5) and (19) are also included in the scheme yielding the debris cross-sectional area $A_{D}$ and its reversed velocity $u_{D}$.

The initial conditions are: $L(0)=L_{0}$, the original length of the projectile; $u(0)=u_{0}$, the impact velocity and $x(0)=0$. A typical solution step involves the following calculations:

- $\quad \dot{u}$ from eqn (4).

- $\quad \dot{x}, A_{D}$ and $u_{D}$ from eqns (5), (18) and (19). Due to the algebraic nonlinearity of these equations, the solution is achieved through an iterative process. The iteration involves $\dot{x}$ only if $\lambda>1$, otherwise iterations are performed only for determining 
$A_{D}$. It should be noted that eqn (5) is applied assuming $\rho_{R} \cong \rho_{D}$. At the end of the process, the obtained values of $\alpha$ and $\lambda$ can be used to calculate the size of the cavity and the radius of the contact area.

- $\quad \dot{L}$ from eqn (3).

The values of the independent variables at step $j+1$ are obtained from those at step $j$ using

$$
u_{j+1}=\dot{u}_{j}(\Delta t)+u_{j}, x_{j+1}=\dot{x}_{j}(\Delta t)+x_{j}, L_{j+1}=\dot{L}_{j}(\Delta t)+L_{j}
$$

and the process is repeated according to the scheme described above.

This algorithm was programmed in FORTRAN. The validity of analysis and its numerical implementation was tested through runs using realistic data and comparisons of its predictions with published experimental and analytical results.

\section{NUMERICAL RESULTS}

\subsection{Penetration versus impact velocity}

The analysis and the program were tested on two cases of medium and high velocity impact among those reported by Hohler and Stilp [13]. The first case involved steel CrV3 projectiles penetrating a high strength steel target. The values of $810 \mathrm{MPa}$ and $1270 \mathrm{MPa}$ for the ultimate tensile stresses of projectile and target material, respectively, were deduced from their respective Brinell numbers of 230 and 360, adopting the approximation proposed by Tabor [16]. The blunt head rod projectile had a mass of $11 \mathrm{~g}$, a diameter of $5.8 \mathrm{~mm}$ and an initial length of $58 \mathrm{~mm}$. The densities of both projectile and target were taken equal to $7,830 \mathrm{~kg} / \mathrm{m}^{3}$. Experimental results for the maximum penetration were available for a range of impact velocities from 570 to $3,700 \mathrm{~m} / \mathrm{s}$.

Numerical results consistent with the experimental data, shown in Fig. 2, were obtained by controlling the values of parameters $a$ and $\beta$. It was found that $a$ needs to be well above 4 , that is, the value identified as appropriate for rigid projectile penetration. Also, $\beta$ needs to be well above unity; this means that the debris dissipated energy makes a substantial contribution to the energy balance.

The results shown in Fig. 2 were obtained for two pairs of $a$ and $\beta$ values, namely $a=6.5$, $\beta=4.5$ and $a=7.5, \beta=3.5$. It is noted that once some rational values of these parameters are identified, the results show little sensitivity to their variation, especially at high impact velocities. The first set of results appear to be in better agreement with experimental results within the middle impact velocity range, that is, from around 1,200 to 2,300 m/s. Within this range, cavitation gradually diminishes until it is only due to the reverse flow of debris. Below an impact velocity of $1,200 \mathrm{~m} / \mathrm{s}$, the analysis does not provide an answer because eqn (18) cannot yield a positive value for $\dot{x}$.

The performance of the model within the medium impact velocity range confirms its validity for the cases when the material resistance plays an important role in penetration control. The calibration of the model based on experimental evidence reveals the significant extent of the debris dissipated energy and thus leads to a better insight into the physical phenomenon.

The second set of experiments involved tungsten sinter alloy D17 projectiles penetrating a high strength steel target. The projectile and target Brinell numbers were given as 290 and 270 , respectively. 


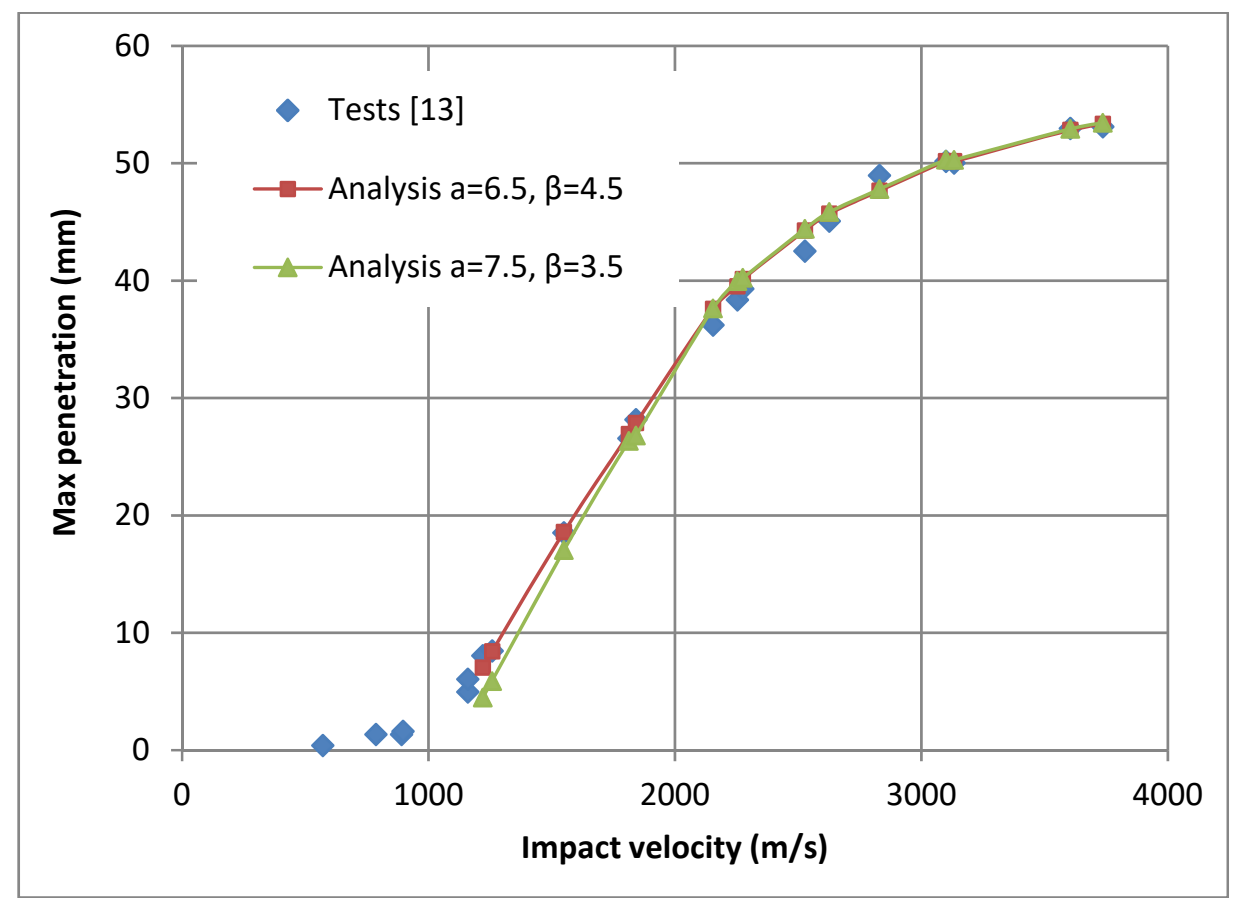

Figure 2: Maximum penetration versus impact velocity in the case of steel CrV3 $(\mathrm{BHN}=230)$ projectiles against high strength steel target $(\mathrm{BHN}=360)$.

From these values, ultimate tensile stresses of 1,024 MPa and $953 \mathrm{MPa}$ were deduced for the projectile and target material, respectively. The blunt head rod projectile had a mass of $26.05 \mathrm{~g}$, a diameter of $5.8 \mathrm{~mm}$ and an initial length of $58 \mathrm{~mm}$. The densities of projectile and target were taken equal to 17,000 and $7,830 \mathrm{~kg} / \mathrm{m}^{3}$, respectively. Experimental results for the maximum penetration were available for a range of impact velocities from 950 to $2,140 \mathrm{~m} / \mathrm{s}$.

The experimental data and the respective numerical predictions of maximum penetration are shown in Fig. 3. In contrast to the first testing programme in which the projectile is softer than the target, in this second set of experiments, the reverse is true. Despite this, it is noted that the values of parameters $a$ and $\beta$ for which reasonably good agreement with test data is achieved are not very different from those of the previous example. The small sensitivity of the numerical results to variations of the $a$ and $\beta$ parameters is again noted. The higher value for $a$ reflects, perhaps, the higher force required by the target material to produce plastic flow in the eroded projectile. The underestimation and overestimation of penetration for the higher and medium impact velocity ranges, respectively, may be attributed to ignoring the dependence of $\beta$ on penetration velocity.

\subsection{Time histories}

The evolution of projectile penetration and rod erosion was observed experimentally by Orphal and Anderson using flash X-rays [14]. In their experiment, a tungsten rod impacted a Zelux target at a velocity of $3,610 \mathrm{~m} / \mathrm{s}$. The projectile had a mass of approximately $0.2 \mathrm{~g}$, a 


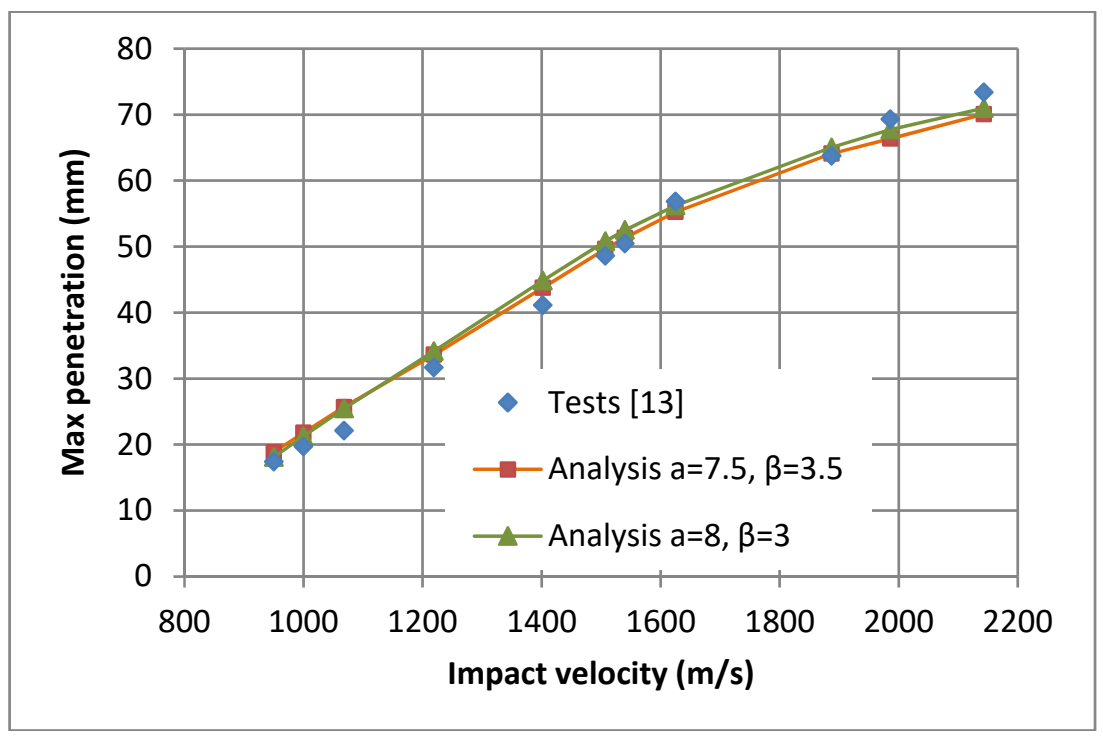

Figure 3: Maximum penetration versus impact velocity in the case of tungsten sinter alloy $(\mathrm{BHN}=290)$ projectiles against high strength steel target $(\mathrm{BHN}=270)$.

diameter of $0.508 \mathrm{~mm}$ and a length of $50.8 \mathrm{~mm}$. Its material was assumed to have a density of $19,300 \mathrm{~kg} / \mathrm{m}^{3}$ and a flow stress of $1,500 \mathrm{MPa}$, which was the value adopted by the authors in their numerical simulations. The target material is a polycarbonate; its density of 1,200 $\mathrm{kg} / \mathrm{m}^{3}$ and tensile strength of $68 \mathrm{MPa}$ were obtained from internet sources [19].

The present analysis was applied to the Orphal and Anderson data with the parameters $a$ and $\beta$ set equal to 8.5 and 2 , respectively, although, at such a high impact velocity, the results show very little sensitivity to their variations. The predicted maximum penetration was $203 \mathrm{~mm}$, which is in perfect agreement with the value given by the shaped charge jet hydrodynamic model according to which [20]

$$
x_{\max }=L_{0}\left(\frac{\rho_{R}}{\rho_{T}}\right)^{1 / 2} .
$$

The target length was $114.3 \mathrm{~mm}$; this means that the projectile exited the target before being fully eroded after travelling through its length over a period of approximately $40 \mu \mathrm{s}$ as shown in Fig. 4(a) and (b). In these figures, a small-time shift of the experimental measurements to the left is observed; this is because the time origin of these results coincides with the first X-ray, which showed the projectile and target prior to impact.

Orphal and Anderson obtained average values for the penetration and the reverse debris flow velocities from their experimental data; these values were $2.72 \pm 0.15 \mathrm{~mm} / \mu \mathrm{s}$ and $2.10 \pm 0.14 \mathrm{~mm} / \mu \mathrm{s}$, respectively. The present analysis produced the time histories of these variables plotted in Fig. 5 for the duration of projectile travel through the target. The average penetration and the reverse debris flow velocities over the first $40 \mu \mathrm{s}$ of analytical output were found equal to $2.85 \mathrm{~mm} / \mu \mathrm{s}$ and $2.26 \mathrm{~mm} / \mu \mathrm{s}$, respectively. These values differ from the experimentally obtained ones by $4.8 \%$ and $7.6 \%$, respectively. 


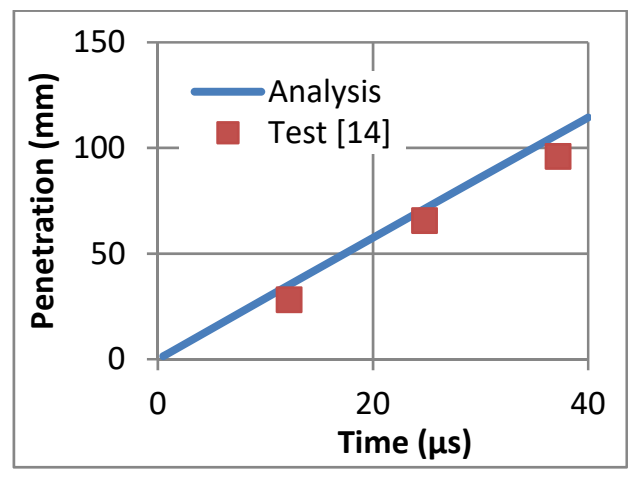

(a)

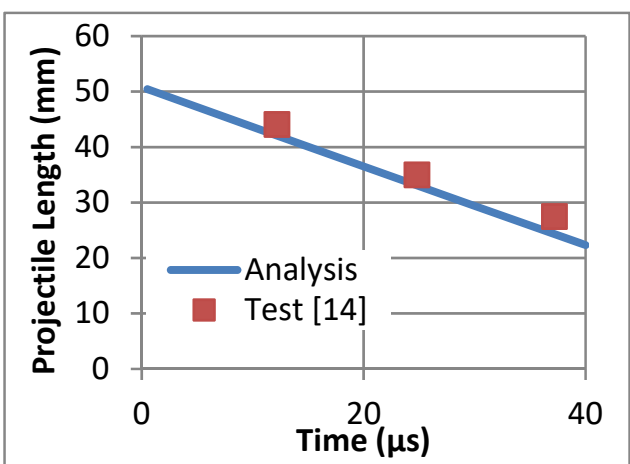

(b)

Figure 4: (a) Penetration depth; and (b) Length of the rod versus time. Tungsten $\operatorname{rod}(f T=$ $1,500 \mathrm{MPa})$ against Zelux target $(f T=68 \mathrm{MPa})$.

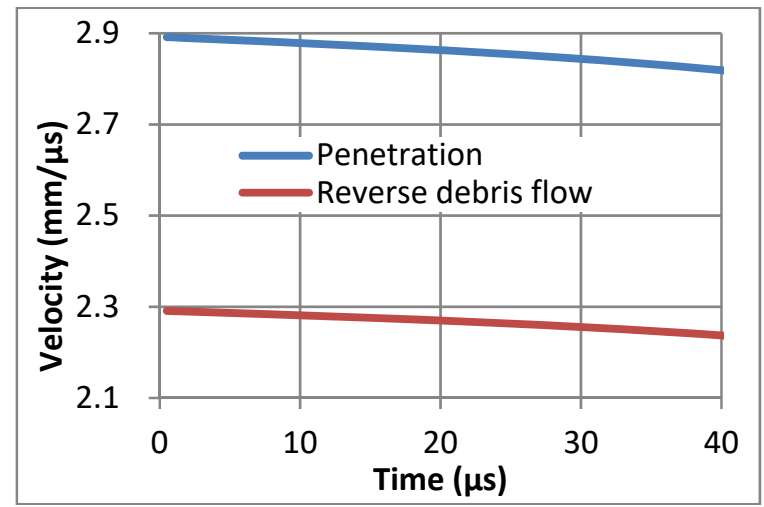

Figure 5: Time histories of penetration and reverse debris velocities for the Orphal and Anderson data [14].

Orphal and Anderson also performed numerical simulations of hypervelocity impacts including that of their own test for which however they adopted a shorter rod of $10.16 \mathrm{~mm}$ length for computational reasons and another polycarbonate having a flow stress of 75.9 $\mathrm{MPa}$, that is, slightly higher than that of Zelux. The maximum cavitation size they predicted was approximately $6 D_{R}$ where $D_{R}$ is the diameter of the rod. The present analysis yielded a cavitation size ranging from $5.87 D_{R}$ to $5.74 D_{R}$ for the first $40 \mu \mathrm{s}$ of penetration. This is another indication of the reliability as well as the wider scope of the proposed model.

\section{DISCUSSION}

Further results were obtained using the data from an earlier experimental programme [9] and a numerical simulation [2]. These results were found consistent with the measured 
penetration rates and the calculated penetration time histories. A more recent test programme [21] generated a large amount of measurements for the maximum penetration of hard projectiles into much softer targets for a wide range of impact velocities. The application of the present analysis to these data confirmed again that there is a lower impact velocity limit below which the model assumptions are not valid and a rigid projectile analysis [8] would provide more reliable results.

In the case of hypervelocity impacts, that is, with striking velocities above $3 \mathrm{~km} / \mathrm{s}$, the strength effects have been found negligible in numerical simulations [2]. Then, hydrodynamic flow conditions prevail and rod projectiles behave like shaped charge jets, which belong to the same projectile category [1] but since they are totally plastic upon impact, their interaction with targets is essentially hydrodynamic while the deformable rod has a rigid non-eroded part during penetration and, as a consequence, material strength also contributes to the process.

As with numerical simulations, the present model also predicted, for very high impact velocities, maximum penetrations close to those given by the hydrodynamic theory governing shaped charge jet penetration. The latter model yielded eqn (20) for maximum penetration, which has been enhanced to include the cratering effect [9]. The predictions from the present system of differential equations are similar to those obtained from eqn (20) as well as a simple hydrodynamic relation for the penetration rate [9].

Jet penetration was predicted by a powerful software code in the context of offshore structure removal by explosive cutting [22]. The present model was also applied to the same event and gave results consistent with those obtained by the computer code. In addition, the model generated the force profile subsequently applied to investigate the consequences of the removal process on structural integrity.

The developed solution algorithm was thus applied to the data from various impact tests and numerical simulations reported in the literature; this helped identifying rational and consistent values for the empirical model so that its predictions were in good agreement with the published results. There is scope for further testing the developed computer code on the multitude of published impact cases ensuring however that the properties of used materials are specified with adequate accuracy. Programming itself could be enhanced to deal more effectively with all types of projectile head shapes, especially at lower impact velocities [23].

\section{CONCLUSIONS}

The work reported in this paper met its main objective, namely, to re-assess and enrich a simple deformable projectile penetration theory using for comparison and calibration experimental evidence and other analytical results. The numerical implementation of the theory allows the fast determination of the shock characteristics, which can be very helpful in structural design against impact. For a wide range of impact velocities, these characteristics depend not only on the impact energy but also on the material properties of the impacting bodies.

The importance of the model lies in its ability to provide answers for the medium impact velocity range within which the projectile is partially eroded when it stops. Satisfactory agreement between analytical and experimental results was achieved in this range with suitable choices of values for $a$ and $\beta$ parameters. With regard to $\beta$, in particular, a degree of dependence on impact energy may be expected but this was accounted for in the model only for penetration rates lower than the cavitation one. For higher velocities, it was considered practical to assume a constant $\beta$ thus preserving the model's simplicity.

The model did not provide answers below a certain velocity limit which depends on the combined projectile-target plastic properties. This is an expected limitation since the 
projectile may be only slightly deformed at velocities within this lower range and thus a rigid projectile analysis [8], [10] may be more relevant.

The model was shown to be valid in the hypervelocity range since its predictions for maximum penetration, penetration rate and reverse debris flow were found consistent with the answers given by the simple hydrodynamic model governing shaped charge jet penetration.

It is important to improve the effectiveness and enhance the scope of the presented analysis without substantially increasing its complexity. Critical examination of the results points to the direction the research should follow. The main effort should focus on a more precise definition of material and geometric parameters and on increasing confidence in the use of available data. An attempt can be made to make the model applicable to oblique impacts within a limited range of angles of incidence. Progress in modelling can be assisted by parallel numerical work based on advanced constitutive theories.

The target material in most engineering applications is considered homogeneous and isotropic but recent designs have introduced more effective combinations of metals. Composite, stratified plates with the capacity of resisting high-energy impacts are mentioned in the literature. These new applications have generated considerable interest among researchers as indicated by the considerable amount of relevant publications. The complexity and diversity of the problem should continue to attract the interest of prospective investigators aiming at developing simple theories that account for the special failure mechanisms observed in these new materials.

\section{REFERENCES}

[1] Backman, M.E. \& Goldsmith, W., The mechanics of penetration of projectiles into targets. International Journal of Engineering Science, 16(1), pp. 1-99, 1978.

[2] Misey, J.J., Analysis of long rod penetration at hypervelocity impact. Report No. 1982, USA Ballistic Research Laboratory, Aberdeen, Maryland, 1977.

[3] Rosenberg, Z. \& Dekel, E., Material similarities in long-rod penetration mechanics. International Journal of Impact Engineering, 25, pp. 361-372, 2001.

[4] Park, B.Y., Leavy, R.B. \& Niederhaus, J.H.J., Penetration of rod projectiles in semiinfinite targets: a validation test for Eulerian X-FEM in ALEGRA. SANDIA Report SAND2013-1863, Sandia National Laboratories, Albuquerque, New Mexico and Livermore, California, 2013.

[5] Rubin, M.B. \& Yarin, A.L., A generalized formula for the penetration depth of a deformable projectile. International Journal of Impact Engineering, 27(4), pp. 387398, 2002.

[6] Lu, Z.C. \& Wen, H.M., On the penetration of high strength steel rods into semi-infinite aluminium alloy targets. International Journal of Impact Engineering, 111, pp. 1-10, 2017.

[7] Wright, T.W. \& Frank, K., Approaches to penetration problems. Impact: Effects of Fast Transient Loadings, ed. A. A. Balkema, Rotterdam, pp. 85-103, 1988.

[8] Syngellakis, S., An improved model for the penetration of a rigid projectile into a ductile target. International Journal of Safety and Security Engineering, 7(1), pp. 7284, 2017.

[9] Christman, D.R. \& Gehring, J.W., Analysis of high-velocity projectile penetration mechanics. Journal of Applied Physics, 37(4), pp. 1579-1587, 1966.

[10] Hill, R., Cavitation and the influence of headshape in attack of thick targets by nondeforming projectiles. Journal of Mechanics and Physics of Solids, 28, pp. 249-263, 1980 . 
[11] Tate, A., A simple hydrodynamic model for the strain field produced in a target by the penetration of a high speed long projectile. International Journal of Engineering Science, 16, pp. 845-858, 1978.

[12] Gobinath, T. \& Batra, R.C., A steady state axisymmetric penetration problem for rigid/perfectly plastic materials. International Journal of Engineering Science, 29, pp. 1315-1331, 1991.

[13] Hohler, V. \& Stilp, A.J., Hypervelocity impact of rod projectiles with L/D from 1 to 32. International Journal of Impact Engineering, 5, pp. 323-331, 1987.

[14] Orphal, D.L. \& Anderson, Jr., C.E., Streamline reversal in hypervelocity penetration. International Journal of Impact Engineering, 23(1), pp. 699-710, 1999.

[15] Johnson, W., Impact Strength of Materials, Edward Arnold: London, 1972.

[16] Tabor, D., The Hardness of Metals, Clarendon Press: Oxford, 1951.

[17] Batra, R.C. \& Wright, T.W., Steady state penetration of rigid perfectly plastic targets. International Journal of Engineering Science, 24(1), pp. 41-54, 1986.

[18] Hill, R., The Mathematical Theory of Plasticity, Oxford University Press: Oxford, 1950.

[19] Westlake plastics. http://www.westlakeplastics.com/product.php?c=EN\&n=56. Accessed on: Mar. 2018.

[20] Mayseless, M. \& Genussov, R., Jet penetration into low density targets. International Journal of Impact Engineering, 23(1), pp. 585-595, 1999.

[21] Forrestal, M.J. \& Piekutowski, A.J., Penetration experiments with 6061-T6511 aluminum targets and spherical-nose steel projectiles at striking velocities between 0.5 and $3.0 \mathrm{~km} / \mathrm{s}$. International Journal of Impact Engineering, 24, pp. 57-67, 2000.

[22] Syngellakis, S. \& Ebdon, R.W., Platform removal by explosive cutting: effect of stress waves on structural integrity. Structural Dynamics: Recent Advances, eds. M. Petyt, H.F. Wolfe \& C. Mei, Elsevier: London, pp. 289-298, 1991.

[23] Khoda-Rahmi, H., Fallahi, A. \& Liaghat, G.H., Incremental deformation and penetration analysis of deformable projectile into semi-infinite target. International Journal of Solids and Structures, 43, pp. 569-582, 2006. 